\title{
Model of a Vascular C-Arm for 3D Augmented Fluoroscopy in Interventional Radiology
}

\author{
S. Gorges ${ }^{1,2}$, E. Kerrien ${ }^{1}$, M-O. Berger ${ }^{1}$, Y. Trousset $^{2}$, J. Pescatore ${ }^{2}$, \\ R. Anxionnat ${ }^{1,3}$, and L. Picard ${ }^{3}$ \\ 1 LORIA-INRIA Lorraine, France \\ 2 GE Healthcare, Buc, France \\ 3 Diagnostic and Therapeutic Neuroradiology Dept, Univ Hosp of Nancy, France \\ Sebastien.Gorges@loria.fr
}

\begin{abstract}
This paper deals with the modeling of a vascular C-arm to generate 3D augmented fluoroscopic images in an interventional radiology context. A methodology based on the use of a multi-image calibration is proposed to assess the physical behavior of the C-arm. From the knowledge of the main characteristics of the $\mathrm{C}$-arm, realistic models of the acquisition geometry are proposed. Their accuracy was evaluated and experiments showed that the C-arm geometry can be predicted with a mean $2 \mathrm{D}$ reprojection error of $0.5 \mathrm{~mm}$. The interest of $3 \mathrm{D}$ augmented fluoroscopy is also assessed on a clinical case.
\end{abstract}

\section{Introduction}

In order to guide tools during the procedure, the interventional radiologist uses a vascular C-arm to acquire 2D fluoroscopy images in real time. Today, 3D X-ray angiography (3DXA) is widely available on modern vascular C-arms. Such 3D images are recognized as being of a daily clinical usefulness for the planning and follow-up of the treatment of cerebral pathologies [1. One next step is to leverage the high-resolution volumetric information provided by 3DXA to complement fluoroscopy images and ease the tool guidance. This requires registering 3DXA with fluoroscopy images, for any orientation of the C-arm.

Image-based registration [2] was investigated to match MRA with Digital Subtracted Angiography (DSA) images. Though providing accurate registration, such methods require the injection of contrast agent for the reference 2D image. Furthermore, their computation time together with the manual interaction necessary to initiate the registration [3] still hampers their wide integration in an ever tighter medical workflow.

Using 3DXA, both images to register are acquired on the same machine. Registration can be deduced from a model of the C-arm, based on the information provided in real-time by the system sensors, such as the C-arm angles. The $a$ priori model in [4] does not accurately fit the acquisition geometry, due to slight mechanical deformations undergone by the C-arm. More sophisticated models have been proposed in [5]6. Though encouraging with regards to the precision of the registration, these works proved that the a priori model was not accurate enough to render the effective mechanical behavior of the C-arm. 
In this paper, an a posteriori model of the C-arm motion is built through a series of measurements relying on vision-based methods. The aim is twofold: on one hand improve the quality of the registration and on a second hand effectively render the mechanical behavior of the $\mathrm{C}$-arm, including mechanical deformations.

\section{The Vascular C-Arm}

\subsection{The C-Arm and Its Sensors}

During a clinical procedure, the C-arm can be oriented in any incidence that the physician reckons as the best suitable for the treatment (Fig. 1). The orientation is classically described by two anatomical angles: $\alpha=$ cranio-caudal (CC) and $\beta$ $=$ right/left anterior orientation $(\mathrm{RAO} / \mathrm{LAO})$. Furthermore, the imager can be translated to adjust its distance to the X-ray tube (Source to Image Distance, or SID). The SID and the $\alpha$ and $\beta$ angles are measured in real time by sensors.

\subsection{The Acquisition Geometry}

The C-arm can be modeled as a pinhole camera by a projection matrix $\mathbf{M}$, relating any $3 \mathrm{D}$ point $X$ to its corresponding projection $q$ in the acquired image:

$$
q=\mathbf{M} X \quad \text { with } \quad \mathbf{M}=\mathbf{I E}=\left[\begin{array}{ccc}
f & 0 & u_{0} \\
0 & f & v_{0} \\
0 & 0 & 1
\end{array}\right][\mathbf{R} \mid \mathbf{T}]
$$

The intrinsic parameters $\mathbf{I}$ describe the projection parameters from the X-ray tube to the $2 \mathrm{D}$ view: $\left(u_{0}, v_{0}\right)$ is the principal point and $f$ is the focal length in pixels (square pixels); the extrinsic parameters $\mathbf{E}$ define the orientation $\mathbf{R}$ and position $\mathbf{T}$ of the acquisition system in a world coordinate system.

\subsection{The Predictive C-Arm Model}

Model-based approaches 45,6] all aim at estimating the intrinsic and extrinsic parameters, thus building up the $\mathbf{M}$ matrix. The intrinsic parameters $\left(u_{0}, v_{0}\right)$ are assumed constant while $f$ directly depends on the SID.

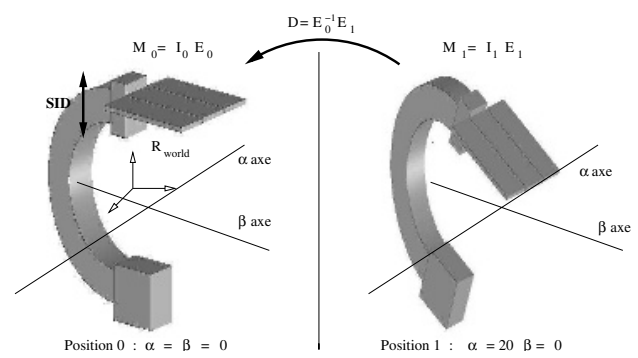

Fig. 1. The vascular C-arm in two different orientations. The change in the acquisition geometry $\mathbf{M}$ is given by the rigid motion $\mathbf{D}$, expressed in the world coordinate frame. 
The extrinsic parameters are assumed to be known in a reference position, which is straightforward. To compute them in a different orientation, the rigid motion $\mathbf{D}$ of the $\mathrm{C}$-arm is modeled as a function of the $\alpha$ and $\beta$ angles (Fig. 1). Dumay 4 modeled $\mathbf{D}$ as made of two independent rotations around the $\alpha$ and $\beta$ axes respectively. Both axes were assumed to be orthogonal and to intersect. Kerrien [5] showed that this does not exactly hold and proposed to calibrate the axes. Cañero [6] compared 4 models with growing complexity, starting from Dumay's model, that significantly improved the accuracy of the registration.

Still, the C-arm bears slight mechanical bendings that impair the accuracy of the previous models. Reliable and independent measurements of intrinsic and extrinsic parameters should help understand these deformations. Next section describes how such measurements can be made.

\section{Robust Estimation of the Acquisition Geometry}

\subsection{Classical Geometric Calibration}

The projection matrix $\mathbf{M}$ is classically estimated through the minimization of the reprojection error $\mathcal{E}_{r}$ on a set of $3 \mathrm{D}$ markers $\left(X_{i}\right)$ :

$$
\mathcal{E}_{r}(\mathbf{M})=\frac{1}{n} \sum_{i=0}^{n}\left\|\mathbf{M} X_{i}-q_{i}\right\|^{2}
$$

where $n$ is the number of detected markers $\left(q_{i}\right)$ in the image. In practice, we use a phantom called "helix phantom" which is made of a hollow Lucite cylinder in which lead markers are inserted according to an helicoidal pattern. Although the estimated projection matrix is known with a sub-pixel reprojection error, its decomposition into intrinsic and extrinsic parameters is known to be unstable.The statistical noise affecting this measurement prevents us from computing independent and reliable intrinsic and extrinsic parameters.

\subsection{Multi-image Calibration}

To reduce the statistical noise, calibration can be repeated with varying extrinsic parameters and fixed intrinsic parameters. Thereby, the inter-dependence between both sets of parameters is reduced, so that reliable intrinsic parameters are estimated. In [7], the camera is moved around the calibration target. In our case, there is no valid reason to believe the intrinsic parameters do not depend on the C-arm orientation. As a result, for a given $\mathrm{C}$-arm orientation, $N$ images of the helix phantom are taken, with the phantom being moved in both rotation and translation between each image acquisition. This step is called multi-image calibration. The common intrinsic parameters $\mathbf{I}$ and $N$ extrinsic parameters $\left(\mathbf{E}_{i}\right)$ are then estimated simultaneously by minimizing the residual reprojection error $\mathcal{R}_{m}$ on the $N$ images, using Levenberg-Marquardt algorithm:

$$
\mathcal{R}_{m}=\frac{1}{N} \sum_{i=0}^{N} \mathcal{E}_{r}\left(\mathbf{M}_{i}\right) \quad \text { with } \quad \mathbf{M}_{i}=\mathbf{I E}_{i}
$$




\subsection{Per-Axis Analysis}

Our medical institution is equipped with a vascular $\mathrm{C}$-arm mounted with the latest generation of flat panel detectors (INNOVA 4100 - GE Healthcare, Buc, France), thus bearing no geometrical distortions. The pixel size is $0.2 \mathrm{~mm}$.

Experimental values showed that 30 images were enough to obtain a stable estimation of the intrinsic parameters. More, bootstrap techniques showed that the precision of the intrinsic parameters was always better than 2.5 pixels.

The Intrinsic Parameters. The multi-image calibration was used to study how the intrinsic parameters varied when the $\mathrm{C}$-arm was rotated around either the $\alpha$ axis or the $\beta$ axis, with a fixed SID $(1180 \mathrm{~mm})$. For each axis, 5 orientations were chosen: $\mathcal{P}_{\alpha}=\left\{(\alpha, \beta) \mid \beta=0 \& \alpha \in\left[-40^{\circ},-20^{\circ}, 0^{\circ}, 10^{\circ}, 20^{\circ}\right]\right\}$ and $\mathcal{P}_{\beta}=$ $\left\{(\alpha, \beta) \mid \alpha=0 \& \beta \in\left[-90^{\circ},-40^{\circ}, 0^{\circ}, 40^{\circ}, 90^{\circ}\right]\right\}$. For each orientation, a multiimage calibration was performed using $N=30$ images.

The focal length remained almost constant whatever the orientation of the C-arm. Figure 2] a shows the measurements made on $\left(u_{0}, v_{0}\right)$, the shorter lines being related to the $\alpha$ rotation. Given the 2.5 pixels precision of the method, no definite change of the intrinsic parameters could be observed except for $u_{0}$ which clearly presents a smooth variation during a rotation around the $\beta$ axis. This imprecision also explains why the curves do not intersect for $\alpha=\beta=0$.

The Extrinsic Parameters. The multi-image calibrations also provide $N$ extrinsic matrices $\mathbf{E}$ for each orientation in the $\mathcal{P}_{\alpha}$ and $\mathcal{P}_{\beta}$ sets (see eq. 1). These parameters are stable, since estimated with stable intrinsic parameters. A subset of images, one per orientation, was taken with a common helix phantom position, thereby providing a subset of extrinsic matrices, one per orientation, expressed in the same coordinate system.

The orientation common to both sets was chosen as the reference position $(\alpha=\beta=0)$. Following [5], the rigid motion $\mathbf{D}$ of the $\mathrm{C}$-arm was computed by
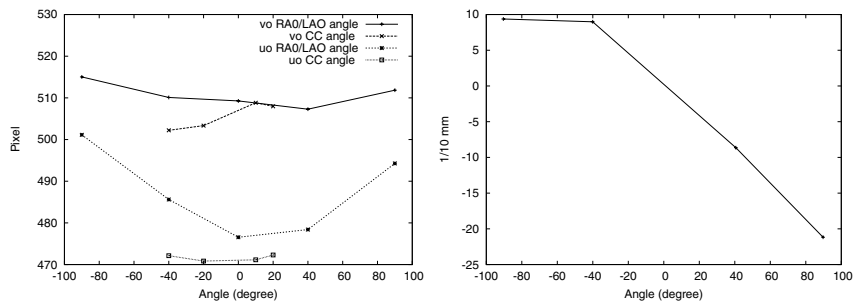

Fig. 2. Mechanical deformations. Left: Variations of $\left(u_{0}, v_{0}\right)$. Right: factor $\lambda$.

Table 1. Per-axis rotation parameters

\begin{tabular}{|c|c|c|c|c|c|c|c|c|}
\hline & \multicolumn{3}{|c|}{$\alpha$} & \multicolumn{4}{|c|}{$\bar{\beta}$} & \\
\hline & $C[\mathrm{x}, \mathrm{y}, \mathrm{z}]$ & $(\mathrm{mm})$ & $\phi\left(^{\circ}\right)$ & & {$[\mathrm{x}, \mathrm{y}, \mathrm{z}]$} & $(\mathrm{mm})$ & $\phi\left(^{\circ}\right)$ & $\theta_{\text {err }}\left({ }^{\circ}\right)$ \\
\hline mec & $\begin{array}{ll}1.75 & 0.53\end{array}$ & -29.04 & 0.48 & {$\left[0.7^{6}\right.$} & 24.86 & -0.04 & 0.06 & 0.26 \\
\hline$\overline{\text { std }}$ & $\begin{array}{ll}0.33 & 0.1\end{array}$ & 1.20 & 0.30 & {$[0.9$} & \begin{tabular}{|l|l}
0 & 0.67
\end{tabular} & 0.003 & 0.02 & 0.18 \\
\hline
\end{tabular}


compositing extrinsic parameters (see also Fig. 1). For analysis purpose, D was expressed as:

$$
\mathbf{D}=\left[\mathbf{R}_{\theta} \mid T\right]=\left[\mathbf{R}_{\theta} \mid\left(\mathbf{I d}-\mathbf{R}_{\theta}\right) C+\Delta T\right]
$$

where $\mathbf{R}_{\theta}$ is a rotation of angle $\theta$ and axis $\nu, C$ is the closest point to the origin on the axis of rotation, and $\Delta T$ is a residual translation, proportional to $\nu: \Delta T=\lambda \nu$.

A per-axis basis analysis of the rigid motions $\mathbf{D}$ is reported in table 3.3 , the stability of the rotation axis is verified through statistics on the position of $C$ and by computing the angular deviation $\phi$ of $\nu$ with respect to its average value. The difference $\theta_{\text {err }}$ observed between the computed $\theta$ and the sensor data is also very small. The norm of $\Delta T$ is below $0.2 \mathrm{~mm}$ for the $\alpha$ axis. On the opposite, it cannot be neglected for the $\beta$ axis: provided that $\Delta T=\lambda \nu$, figure 2 displays the variation of $\lambda$ according to the angle $\beta$. As a conclusion, the rotation model is valid for the $\mathrm{C}$-arm motion around the $\alpha$ axis, but not for $\beta$ which requires a further translation parallel to the rotation axis.

\subsection{Conclusion}

The multi-image calibration is an accurate method to determine reliable intrinsic and extrinsic parameters. A per-axis analysis unveiled a variation of the $u_{0}$ intrinsic parameter and an important residual translation during a $\beta$ rotation. These may be interpreted as respectively a physical change of the relative position between the X-ray tube and the flat panel, and a global mechanical bending of the C-arm under its own weight in lateral positions. Such effects are taken into account in two models described in the next section.

\section{Predictive Models of the C-Arm Acquisition Geometry}

\subsection{Description}

The aim in modeling the C-arm acquisition geometry is to be able to predict, for any orientation $(\alpha, \beta)$ of the $\mathrm{C}$-arm, the acquisition geometry:

$$
\mathbf{M}_{\alpha, \beta}=\mathbf{I}_{\alpha, \beta} \mathbf{E}_{\alpha, \beta}
$$

The extrinsic parameters $\mathbf{E}_{\alpha, \beta}$ are recovered by modeling the rigid motion imparted to the $\mathrm{C}$-arm to move from a reference orientation $(\alpha=\beta=0)$ to $(\alpha, \beta)$. Under the hypothesis that the $\alpha$ and $\beta$ motions are independent, this rigid motion is a composition of two rigid motions: $\mathbf{D}_{\alpha}$ around the $\alpha$ axis, and $\mathbf{D}_{\beta}$ around the $\beta$ axis:

$$
\mathbf{E}_{\alpha, \beta}=\mathbf{E}_{0} \mathbf{D}_{\alpha} \mathbf{D}_{\beta}
$$

where $\mathbf{E}_{0}=\left[\mathbf{R}_{0} \mid T_{0}\right]$ are the extrinsic parameters in the reference orientation.

According to the above measurements, $\mathbf{D}_{\alpha}$ is a rotation of angle $\alpha$, read on the sensor, and parameterized by an axis vector $\nu_{a}$ and a fixed point $C_{a}$. $\mathbf{D}_{\beta}$ is a rotation of angle $\beta$, read on the sensor, and parameterized by an axis 
Table 2. Reprojection error in pixels for the matrices predicted by both models. The mean error for model $\mathcal{M}_{f}$ was 2.37 pixels $(\operatorname{std}=1.48)$. The mean error for model $\mathfrak{M}_{m}$ was 2.31 pixels $(\mathrm{std}=1.31)$. The pixel size is $0.2 \mathrm{~mm}$.

\begin{tabular}{|c||c|c|c|c|c|c|c|c|c|c|c|c|c|c|c|}
\hline$\alpha\left(^{\circ}\right)$ & -28.8 & -28.8 & -28.8 & -8.8 & -8.8 & -8.8 & -8.8 & -8.8 & -0.3 & -0.3 & -0.3 & -0.3 & -0.3 & 19.1 & 19.1 \\
\hline$\beta\left(^{\circ}\right)$ & -40.4 & 1.3 & 40 & -90.4 & -39.1 & 0.1 & 41.1 & 79.8 & -90.4 & -39.6 & 0.4 & 41.2 & 80.3 & -19.3 & 0.1 \\
\hline \hline $\mathcal{M}_{f}$ & 5.81 & 3.43 & 5.61 & 1.93 & 1.90 & 2.24 & 2.59 & 2.24 & 1.20 & 1.27 & 1.28 & 1.38 & 1.60 & 1.83 & 1.25 \\
\hline $\mathcal{M}_{m}$ & 3.63 & 3.44 & 6.10 & 1.47 & 1.72 & 2.25 & 2.26 & 3.20 & 1.59 & 1.39 & 1.28 & 1.26 & 1.66 & 2.19 & 1.25 \\
\hline
\end{tabular}

vector $\nu_{b}$ and a fixed point $C_{b}$ associated to a translation along $\nu_{b}$ of amplitude $\lambda$. Provided the general function shape in figure 2 $\mathrm{b}$, second- and third-order polynomials were tested to model the parameter $\lambda$ as a function of $\beta$. The latter gave better results: $\lambda=\sum_{i=0}^{3} \lambda_{i} \beta^{i}$.

The intrinsic parameter $v_{0}$ is constant, and the focal length $f$ only depends on the SID. The models are described considering a fixed SID, allowing the assumption that $f$ is also constant. According to the above experiments, $u_{0}$ varies as a function of $\beta$. Figure 2, a suggests the shape of a low-order polynomial. Again third-order polynomial proved best: $u_{0}=\sum_{i=0}^{3} \mu_{i} \beta^{i}$.

Thereby a model was built to render the mechanical properties of the C-arm. This model, denoted $\mathcal{M}_{m}$, is parameterized by the vector:

$$
\phi_{m}=\left\{\mathbf{R}_{0}, T_{0} ; v_{a}, C_{a} ; v_{b}, C_{b},\left(\lambda_{i}\right)_{i=0 . .3} ;\left(\mu_{i}\right)_{i=0.3}, v_{0}, f\right\}
$$

A second model was considered, only differing from $\mathcal{M}_{m}$ by considering $u_{0}$ is also constant, thereby assuming intrinsic parameters are constant. The model is denoted by $\mathcal{M}_{f}$ and is parameterized by:

$$
\phi_{f}=\left\{\mathbf{R}_{0}, T_{0} ; v_{a}, C_{a} ; v_{b}, C_{b},\left(\lambda_{i}\right)_{i=0.3} ; u_{0}, v_{0}, f\right\}
$$

\subsection{Calibrating the Models}

Model $\mathcal{M}_{\boldsymbol{f}}$. Since intrinsic parameters are constant, a classical multi-image calibration can be performed, with a moving C-arm and a fixed helix phantom as in [7]. One image was acquired for every orientation in $\mathcal{P}_{\alpha}$ and $\mathcal{P}_{\beta}$. The only difference with section 3.2 is that the $N$ extrinsic parameters are replaced by the components of $\phi_{f}$ modeling the extrinsic parameters. Images of the fixed helix phantom were also taken for C-arm orientations outside $\mathcal{P}_{\alpha}$ and $\mathcal{P}_{\beta}$ for validation purpose. The orientations of this test set are provided in table 2

Model $\mathcal{M}_{\boldsymbol{m}}$. Due to the varying intrinsic parameter $u_{0}$, one multi-image acquisition has to be made for each orientation in $\mathcal{P}_{\alpha}$ and $\mathcal{P}_{\beta}$. These come in addition to the same acquisition as for $\mathcal{M}_{f}$, necessary to manage the extrinsic parameters. This results in the same type of acquisition as made in section 3.3 to study the extrinsic parameters. Again, a global cost function could be designed and minimized but the relatively small influence of the intrinsic parameters compared to that of the extrinsic parameters leads to poorly optimized intrinsic parameters. As often in numerical optimization, we found the adequate cost function to be the weighted sum of two residuals:

$$
\mathcal{R}=\mathcal{R}_{f}+\gamma \sum_{(\alpha, \beta) \in \mathcal{P}_{\alpha} \cup \mathcal{P}_{\beta}} \mathcal{R}_{m}\left(\mathbf{M}_{\alpha, \beta}\right)
$$


where $\mathcal{R}_{f}$ is the residual of the classical multi-image calibration as for the $\mathcal{M}_{f}$ model, and $\mathcal{R}_{m}$ is the residual of our multi-image calibration, as described in section 3.2. $\gamma$ was fixed at 1000, to balance the influence of both terms on $\mathcal{R}$.

\section{$5 \quad$ Validation - Application to 3D Augmented Fluoroscopy}

The limitations of fluoroscopy are well known: contrast medium has to be injected repetitively to visualize the vessels, its image quality is reduced compared to DSA, and finally, it does not provide 3D information. The real-time superimposition of fluoroscopic images with pre-operative 3DXA images could potentially overcome some of these limitations. We call this clinical application " $3 \mathrm{D}$ augmented fluoroscopy". The various validation studies that follow were targeted to such an application. As a result, the reprojection error was chosen as the figure of merit to evaluate our models.

\subsection{Comparison of the Models}

Both $\mathcal{M}_{f}$ and $\mathcal{M}_{m}$ models where calibrated as described above. For each orientation $(\alpha, \beta)$ in the test set, the matrix $\mathbf{M}_{\alpha, \beta}$ predicted by each model was built up and its reprojection error computed. Results are reported in table 2 .

Each model presents a mean reprojection error of about 2.5 pixels which represents $0.5 \mathrm{~mm}$ of error in the image plane (pixel size $=0.2 \mathrm{~mm}$ ). In both cases, the error was below this average error in $84 \%$ of the test orientations. This precision is sufficient for many medical applications and in particular for 3D augmented fluoroscopy. No major differences could be noted between the models. Indeed, in $\mathcal{M}_{f}$, nothing prevents the coupling effect between intrinsic and extrinsic parameters from counteracting the error made when assuming $u_{0}$ is constant.

\subsection{Evaluation of 3D Augmented Fluoroscopy}

Phantom experiment. A silicon phantom of the cerebral vasculature was injected with a contrast medium and a 3DXA was acquired. Then, one fluoroscopy image was taken for each test orientation in table 2. 3D augmented views were

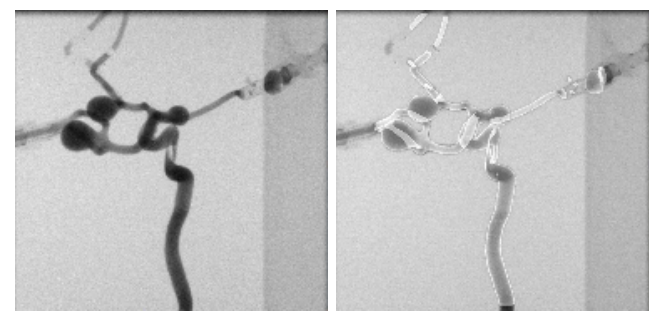

Fig. 3. 3D augmented fluoroscopy on phantom dataset. Left: fluoroscopic image of the phantom; right: superposition of the 3DXA onto the fluoroscopic image. 

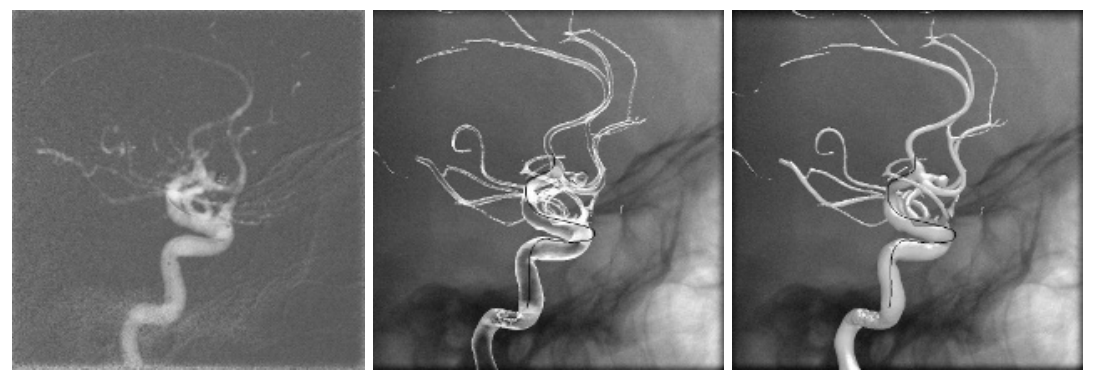

Fig. 4. 3D augmented fluoroscopy on a clinical case with model $\mathcal{M}_{m}$ : Comparison of contrast-enhanced fluoroscopy (left) and 3D augmented fluoroscopy width blending (middle) and surface (right) views. Only the main vessels are shown and the guide wire was manually overlined in black.

generated, using model $\mathcal{M}_{m}$, to allow a visual assessment of the local reprojection error to complement the above global statistics. In Fig 5.2 an augmented image is shown for $\alpha=8.8^{\circ}$ and $\beta=41.1^{\circ}$. The precision of this position is $0.45 \mathrm{~mm}$ (see table 2) which, from a visual standpoint, corresponds to a perfect fit.

Patient Data. A patient underwent an endovascular treatment for an aneurysm. A 3DXA was acquired and fluoroscopy images were captured under an oblique orientation $\left(\alpha=2^{\circ}, \beta=-82^{\circ}\right)$ while the micro-catheter was moved up to the aneurysm. The SID $(1070 \mathrm{~mm})$ used during the procedure was different from the SID used to calibrate the models $(1180 \mathrm{~mm})$. Therefore, the focal length given by the models was updated as $f+(1070-1180) / s_{p}$ where $s_{p}=0.2 \mathrm{~mm}$ is the pixel size.

Visual assessment of 3D augmented views indicated a very accurate match on the region of medical interest, i.e. around the aneurysm. Compared to fluoroscopic images (Fig 5.2 left), the augmented images (Fig 5.2 middle \& right) present a higher image quality and ease the assessment of the tool position within the $3 \mathrm{D}$ vascular anatomy in real time. Furthermore, the surface view (Fig 5.2 right) allows to better analyze superimpositions of vascular structures and can dramatically help the radiologist to understand the vascular bifurcations. Thus, 3D augmented fluoroscopy can make micro-catheter navigation and tool deployment easier.

\section{Conclusion}

A robust calibration method for the intrinsic and extrinsic parameters of a vascular C-arm was proposed and evaluated. Thereby, some mechanical characteristics of the C-arm were assessed and realistic models were built, including slight deformations of the system. These models were evaluated as being able to predict the acquisition geometry for any $\mathrm{C}$-arm orientation with a mean $2 \mathrm{D}$ reprojection error of $0.5 \mathrm{~mm}$. This accuracy enables many medical applications such as 3D augmented fluoroscopy. A clinical case showed that 3D augmented fluoroscopy has the potential to facilitate the classical navigation of the radiologists. 


\section{References}

1. R. Anxionnat, S. Bracard, X. Ducrocq, Y. Trousset, L. Launay, E. Kerrien, M. Braun, R. Vaillant, F. Scomazzoni, A. Lebedinsky and L. Picard. Intracranial Aneurysms : Clinical Value of 3D Digital Subtraction Angiography in the Therapeutic Decision and Endovascular Treatment. In Radiology, (218):799-808, 2001.

2. J.H. Hipwell, G.P. Penney, R.A. McLaughlin, K. Rhode, P. Summers, T.C. Cox, J.V. Byrne, A. Noble, and D.J. Hawkes. Intensity-based 2D-3D registration of cerebral angiograms. In IEEE Trans. on Med. Imag., 22(11):1417-1426, 2003.

3. J.V. Byrne, C. Colominas, J.H. Hipwell, T.C. Cox, A. Noble, G.P. Penney, and D.J. Hawkes. Assessment of a technique for 2D-3D registration of cerebral intra-arterial angiography. In The British Journal of Radiology, 77:123-128, 2004.

4. A. Dumay, J. Reiber and J. Gerbrands. Determination of optimal angiographic viewing angles: Basic principles and evaluation study. In IEEE Trans. on Med. Imag., 13(1):13-24, 1994.

5. E. Kerrien, R. Vaillant, L. Launay, M-O. Berger, and E. Maurincomme. Machine precision assessment for $3 \mathrm{D} / 2 \mathrm{D}$ digital subtracted angiography images registration. In SPIE Medical Imaging'98, San Diego, USA, February 1998.

6. C. Cañero, E. Nofrerías, J. Mauri, and P. Radeva. Modelling the acquisition geometry of a C-arm angiography system for 3D reconstruction. In Conferencia Catalana d'Inteligència Artificial - CCIA'2002, 322-335, 2002.

7. J.M. Lavest, M. Viala and M. Dhome. Do we really need an accurate calibration pattern to achieve a reliable camera calibration. In European Conference on Computer Vision - ECCV'98, Freiburg, Germany,Vol 1, 158-174, 1998. 